\title{
artigo
}

Araújo, M.L.L.M.; Costa, M.J.M.; Brandão, S.A.S.M.; Costa, F.V.B.; Moreira-Araújo, R.S.R.;

Obesidade e excesso de peso em pré-escolares: associação com o consumo de alimentos

\section{Obesidade e excesso de peso em pré-escolares: associação com o consumo de alimentos}

\author{
Obesity and overweight in pre-school: association with food consumption \\ Obesidad y exceso de peso en preescolares: asociación con el consumo de alimentos
}

\begin{abstract}
RESUMO
A pesquisa objetivou identificar a prevalência de excesso de peso e obesidade e sua relação com o consumo de alimentos em pré-escolares da rede privada. Foi do tipo transversal, descritiva, tendo como amostra, 403 pré-escolares. Realizou-se avaliação antropométrica para obtenção do IMC, foram coletadas dados da frequência do consumo de alimentos. Na análise estatística, foi utilizado o teste t de Student, o teste do 2 (Qui-quadrado) e Kruskal-Wallis, com nível de significância de 5\%. Foi utilizada a correlação de Pearson entre o consumo de alimentos e o IMC para a idade. Os resultados demonstraram que $60,1 \%$ das crianças estavam eutróficas e 1,2\% com magreza. Foi constatado risco de excesso de peso, excesso de peso e obesidade de $20,8 \%, 5,2 \%$ e $12,7 \%$, respectivamente. Assim, o consumo de determinados alimentos apresentou forte correlação com o IMC para a idade referente ao risco de excesso de peso, excesso de peso e obesidade.
\end{abstract}

DESCRITORES: Pré-escolares; Consumo alimentar; Obesidade.

\section{ABSTRACT}

The research aimed to identify the prevalence of overweight and obesity and its relationship with food consumption in pre-school children from the private network. It was cross-sectional, descriptive, with a sample of 403 preschoolers. An anthropometric assessment was performed to obtain the BMI, data on the frequency of food consumption were collected. In the statistical analysis, Student's t test, the 2 test (Chi-square) and Kruskal-Wallis were used, with a significance level of $5 \%$. Pearson's correlation between food consumption and BMI for age was used. The results showed that $60.1 \%$ of the children were eutrophic and $1.2 \%$ were thin. The risk of overweight, overweight and obesity was found to be $20.8 \%, 5.2 \%$ and $12.7 \%$, respectively. Thus, the consumption of certain foods showed a strong correlation with BMI for age regarding the risk of overweight, overweight and obesity.

DESCRIPTORS: Preschoolers; Food consumption; Obesity.

\section{RESUMEN}

La investigación tuvo como objetivo identificar la prevalencia de sobrepeso y obesidad y su relación con el consumo de alimentos en niños en edad preescolar de escuelas privadas. Fue transversal, descriptivo, con una muestra de 403 preescolares. Se realizó una evaluación antropométrica para obtener el IMC, se recolectaron datos sobre la frecuencia de consumo de alimentos. En el análisis estadístico se utilizó la prueba t de Student, la prueba de la 2 (Chi-cuadrado) y Kruskal-Wallis, con un nivel de significancia del 5\%. Se utilizó la correlación de Pearson entre el consumo de alimentos y el IMC para la edad. Los resultados mostraron que el $60,1 \%$ de los niños eran eutróficos y el 1,2\% eran delgados. Se encontró que el riesgo de sobrepeso, sobrepeso y obesidad era del $20,8 \%, 5,2 \%$ y $12,7 \%$, respectivamente. Así, el consumo de determinados alimentos mostró una fuerte correlación con el IMC para la edad en cuanto al riesgo de sobrepeso, sobrepeso y obesidad.

DESCRIPTORES: Niños en edad preescolar; Consumo de comida; Obesidad.

RECEBIDO EM: 06/04/2021 APROVADO EM: 30/04/2021

\section{Maria Lícia Lopes Morais Araújo}

Doutoranda do Programa de Pós- Graduação em Alimentos e Nutrição da Universidade Federal do Piauí. Mestra em Ciências e Saúde - na área Métodos Diagnósticos e Análise de Condições de Saúde.

ORCID: 0000-0002-1038-1404 


\section{Mara Jordana Magalhães Costa}

Doutora em Saúde Pública - Faculdade de Saúde Pública da USP, na linha de pesquisa de Saúde Pública e Envelhecimento. Mestra em Ciências e Saúde pela Universidade Federal do Piauí.

ORCID: 0000-0003-2185-3814

\section{Socorro Adriana de Sousa Meneses Brandão}

Doutoranda em enfermagem pela Universidade Federal do Piauí. Mestra em Ciências e Saúde pela Universidade Federal do Piauí. Especialista em Vigilância Sanitária e Epidemiológica pela UNAERP. Especialista em Saúde Pública pela Universidade Federal do Piaúi.

ORCID: 0000-0002-6711-3515

\section{Francisca Vanessa Barros Costa}

Mestra em Ciências e Saúde pela Universidade Federal do Piauí. Especialista em Fisioterapia Hospitalar pela Universidade Estadual do Piauí. Especialista em Neonatologia e Pediatria pela Universidade Estadual do Piauí.

ORCID: 0000-0002-3350-7591

\section{Regilda Saraiva dos Reis Moreira- Araújo}

Pós-Doctor em Ciência dos Alimentos na Faculdade de Farmácia da UFMG e Pós-Doctor em Nutrição em Saúde Pública na FSP/USP. Doutora em Ciência de Alimentos pela Universidade de São Paulo (USP). Mestra em Tecnologia de Alimentos pela Universidade Federal do Ceará (UFC).

ORCID: 0000-0002-3669-2358

\section{INTRODUÇÃO}

A saúde na infância é preditiva da saúde ao longo da vida, constituindo nos primeiros anos, os alicerces sobre os quais se constrói a realização e o potencial em saúde. A adequação da nutrição nos primeiros anos de vida é fundamental para o desenvolvimento e crescimento saudáveis ${ }^{1}$.

Nessa fase as crianças que estão obesas poderão permanecer como tal na fase adulta, tornando-se portadoras potenciais de doenças cardiovasculares, hipertensão arterial sistêmica, dislipidemia aterogênica, apneia do sono, aterosclerose precoce, diabetes e doença hepática gordurosa não alcoólica².

A escola é um espaço favorável para a educação em saúde e na construção de ações no âmbito preventivo, com foco na qualidade de vida, principalmente em territórios vulneráveis ${ }^{3}$.

A Pesquisa Nacional sobre Demografia e Saúde (PNDS, 2006), avaliou crianças até cinco anos de idade e mostrou uma prevalência nacional de excesso de peso de $6,6 \%$, e de $8,8 \%$ na região $\mathrm{Sul}^{4}$.

A POF de 2008-2009, apontou para um aumento na prevalência de excesso de peso no Brasil que atingia $33,5 \%$ das crianças com idade entre cinco e nove anos, va-
A saúde na infância é preditiva da saúde ao longo da vida, constituindo nos primeiros anos, os alicerces sobre os quais se constrói a realização e o potencial em saúde. A adequação da nutrição nos primeiros anos de vida é fundamental para o desenvolvimento e crescimento saudáveis riando de 32\% a $40 \%$ nas regiões Sudeste, Sul e Centro-Oeste e, de 25\% a 30\% nas regiões Norte e Nordeste 5 .

Portanto, torna-se de primordial importância a avaliação do padrão alimentar e a adequação das necessidades nutricionais de crianças em idade pré-escolar, face à relevância da composição da dieta infantil para a manutenção de um estado nutricional adequado. Doenças carenciais estão epidemiologicamente relacionadas à faixa etária em que a criança se encontra. Por outro lado, hábitos alimentares errôneos contribuem para o acréscimo do excesso de peso e obesidade.

Estudos anteriores nessa região mostraram haver associação estatisticamente significativa, entre a anemia e as variáveis de consumo alimentar e, os indicadores antropométricos $\mathrm{P} / \mathrm{I}$ e A/I em pré-escolares de Teresina-Piauí, com baixa ingestão de ferro, proteínas, energia, cálcio e vitaminas, e teores de vitaminas $\mathrm{C}$, acima das recomendações diárias ${ }^{7}$.

Diante do exposto, realizou-se essa pesquisa objetivando conhecer a prevalência de excesso de peso, obesidade e sua correlação com a frequência de consumo de alimentos, em nível escolar e domiciliar, em pré-escolares assistidos em escola privada, de Teresina- Piauí. 


\section{MÉTODOS}

Trata-se de um Estudo Transversal Descritivo de base escolar, que foi realizado em escola da rede particular de ensino da cidade de Teresina-PI, entre o período de março a junho de 2013 com 403 crianças na faixa etária de 2 a 5 anos de idade, em escolas da rede particular de ensino da cidade de Teresina (PI). A pesquisa teve como objetivo verificar a prevalência de excesso de peso, obesidade e relacionar ao consumo alimentar de pré-escolares, a fim de delinear em nosso meio a relação do estado nutricional e o consumo de alimentos.

A escola privada sorteada atendia crianças de diferentes zonas da cidade. $\mathrm{O}$ cálculo da amostra para a estimativa de proporções considerou $10 \%$ no tocante ao excesso de peso e a obesidade, tendo em vista o relato da literatura ${ }^{8}$.

Os pré-escolares foram selecionados de forma "probabilística", sendo que todas as 1.044 crianças matriculadas na Educação Infantil tiveram a chance de participar da pesquisa. Foi utilizada uma lista de números randomizados para identificar cada criança. De cada série foi sorteada uma amostra de 110 pré-escolares, obtendo-se 440 pré-escolares. Quadro 1.

Destes 440 pré-escolares, no final do estudo a amostra foi composta por 403 crianças, tendo em vista que muitos deles faltaram às aulas nos dias da avaliação, havendo perda de $8,41 \%$ da amostra.

Foram excluídas do estudo, crianças com alguma restrição física, motora ou neurológica pudesse que interferir na coleta dos dados antropométricos. Sendo assim, foram excluídas da pesquisa duas crianças, que os pais autorizaram partici-
Os pré-escolares

foram selecionados

de forma

"probabilística",

sendo que todas

as 1.044 crianças

matriculadas na

Educação Infantil

tiveram a chance

de participar da

pesquisa. Foi utilizada

uma lista de números

randomizados para

identificar cada

criança. De cada série

foi sorteada uma

amostra de 110 pré-

escolares, obtendo-se

440 pré-escolares.
Quadro 1.Demonstrativo de número de alunos que entraram para sorteio por série na escola privada de Teresina-PI, 2014.

\begin{tabular}{|l|l|}
\hline SÉRIE & QUANTIDADE DE ALUNOS \\
\hline MATERNAL I & 110 alunos \\
\hline MATERNAL II & 110 alunos \\
\hline INFANTIL I & 110 alunos \\
\hline INFANTIL II & 110 alunos \\
\hline Fonte: Dados da Pesquisa. \\
\hline
\end{tabular}

par da pesquisa, porém se enquadram no grupo de exclusão, uma por possuir paralisia cerebral e outra por ser anã, grau severo.

A pesquisa foi autorizada, por meio de um Termo de Consentimento Institucional e o estudo foi aprovado pelo Comitê de Ética em Pesquisa com seres humanos, da Universidade Federal do Piauí com o CAAE 01140312000005214.

A primeira etapa foi de esclarecimento, para a direção e para os professores, sobre os procedimentos que seriam realizados e os objetivos do estudo. Na segunda etapa, foram enviadas circulares aos pais, mostrando as razões da pesquisa com os devidos objetivos e o Termo de Consentimento Livre e Esclarecido (TCLE) para caso aceitassem que o filho (a) participasse da pesquisa. A terceira etapa foi a coleta de dados das crianças cujo termo de Consentimento Livre e Esclarecido estivesse devidamente assinado pelos pais dos alunos.

A aferição antropométrica seguiu normas padronizadas. Foram mensuradas as variáveis: peso, utilizando uma balança tipo plataforma (até 150 quilogramas e precisão de $0,1 \mathrm{~kg}$ ) aferida previamente; e estatura, com fita antropométrica com escala de $2 \mathrm{~m}$, fixa a uma parede plana e piso sem declínio. O Índice de Massa Corporal (IMC) para criança foi determinado pelo programa Epi-Info 6.049. $\mathrm{O}$ excesso de peso e obesidade foram classificadas de acordo com os pontos de corte, ajustados à idade e gênero, conforme estudo anterior ${ }^{10}$.

O consumo alimentar foi avaliado por meio de um Questionário de Frequência de Consumo de Alimentos (QFCA), validado em estudo anterior ${ }^{11}$. Para análise estatística, foi criado um banco de dados no Programa Estatístico Statistical Package for the Social Sciences (SPSS), versão 13.0. Para verificar a diferença entre as médias das idades entre os sexos foi utilizado o teste $t$ de Student e para verificar a associação entre as variáveis nominais teste do Qui-quadrado. O nível de significância estatística adotado foi de $5 \%(\mathrm{p}<0,05)^{12}$. Para verificar a existência de homogeneidade da população foi utilizado Bartlett test $(\mathrm{p}=0873)$ que mostrou uma distri- 
Tabela 1-Média e desvio padrão das variáveis peso ao nascer, peso atual e altura atual, segundo o sexo das crianças pesquisadas. Teresina, Piauí, 2013.

\begin{tabular}{|c|c|c|c|c|c|c|c|c|c|}
\hline \multirow{3}{*}{ VALORES } & \multicolumn{9}{|c|}{ VARIÁVEIS } \\
\hline & \multicolumn{3}{|c|}{$\begin{array}{c}\text { PESO AO NASCER (KG) ESTATÍSTICA: } \\
\text { TESTE T DE STUDENT=3,296 } \\
\text { P=0,001 }\end{array}$} & \multicolumn{3}{|c|}{$\begin{array}{c}\text { PESO ATUAL (KG) ESTATÍSTICA: } \\
\text { TESTE T DE STUDENT=2,999 } \\
\text { P=0,003 }\end{array}$} & \multicolumn{3}{|c|}{$\begin{array}{l}\text { ALTURA ATUAL (CM) ESTATÍSTICA: } \\
\text { TESTE T DE STUDENT=2,423 } \\
\text { P=0,016 }\end{array}$} \\
\hline & MASCULINO & FEMININO & GERAL & MASCULINO & FEMININO & GERAL & MASCULINO & FEMININO & GERAL \\
\hline Média & 3,294 & 3,113 & 3,202 & 19,102 & 17,782 & 18,427 & 105,0 & 103,0 & 104,0 \\
\hline $\begin{array}{l}\text { Desvio padrão } \\
\text { Fonte: Dados da pesquisa }\end{array}$ & 0,8 & 0,7 & 0,5 & 1,7 & 1,9 & 1,5 & 0,1 & 0,1 & 0,1 \\
\hline
\end{tabular}

buição normal no universo de 403 crianças pesquisadas com $95 \%$ de confiança.

\section{RESULTADOS}

A maioria das crianças pesquisadas, $51,1 \%$ (206) era do sexo feminino, enquanto $48,9 \%$ (197) pertencia ao sexo masculino. Foi feita a média e desvio padrão das variáveis do peso ao nascer, peso atual e altura atual, segundo o sexo das crianças. Dados do estudo referente ao

peso ao nascer, peso atual e altura atual encontram-se na Tabela 1.

Para avaliar o estado nutricional foram utilizados os seguintes índices: Altura para Idade e o IMC para Idade. O índice altura para idade mostrou que $97,8 \%$ da população estudada estava dentro da normalidade e apenas 2,2\% das crianças tinha baixa estatura para idade. Tabela 2.

O IMC para idade mostrou que $60,1 \%$ (242) das 403 crianças pesquisadas estavam eutróficas. Os dados mostraram também

Tabela 2. Frequência do estado nutricional segundo o Índice altura para idade das crianças pesquisadas. Teresina, Piauí, 2013.

\begin{tabular}{lccc} 
VALORES CRÍTICOS & DIAGNÓSTICO NUTRICIONAL & \multicolumn{2}{c}{ FREQUÊNCIA } \\
< Escore-z-3 & Muito baixa estatura para idade & - & N \\
$\geq$ Escore-z-3 e < Escore-z -2 & Baixa estatura para idade & 09 & 2,2 \\
$\geq$ Escore-z -2 & Estatura adequada para idade & 394 & 97,8 \\
Total & & 403 & 100,0 \\
Fonte: Dados da Pesquisa. & & &
\end{tabular}

Tabela 3. Frequência do estado nutricional segundo o IMC para Idade das crianças pesquisadas. Teresina, Piauí, 2013.

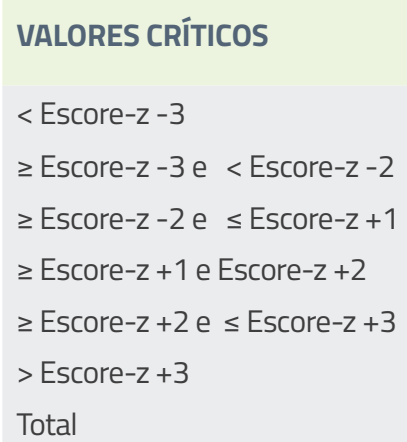

Fonte: Dados da Pesquisa. que 20,8\% (84) dos pré-escolares apresentavam risco de excesso de peso, enquanto $5,2 \%$ (21) tinham excesso de peso e $12,7 \%$ (51) obesidade. Conforme na Tabela 3.

$\mathrm{Na}$ presente pesquisa foi constatado risco de excesso de peso, excesso de peso e obesidade de $20,8 \%, 5,2 \%$ e $12,7 \%$, respectivamente (Tabela 3). Demonstrando uma elevada porcentagem de risco de excesso de peso e obesidade das crianças estudadas.

$\mathrm{Na}$ análise entre o sexo e o estado nutricional, os resultados mostraram que $13,7 \%$ dos meninos apresentaram obesidade e $23,3 \%$, risco de excesso de peso. Enquanto que $11,6 \%$ das meninas apresentaram obesidade e $18,4 \%$ de risco de excesso de peso. Apenas entre os eutróficos houve diferença significativa $(p=0,009)$ entre meninos e meninas. Mostrando que a maioria das crianças eutróficas estava entre o sexo feminino. As crianças com excesso de peso praticamente não tiveram diferença, o mesmo acontecendo com a magreza. Figura 1.

$\mathrm{Na}$ população da presente pesquisa observa-se a prevalência de meninas eutróficas, $63,7 \%$ para $56,3 \%$, dos meninos. Para esse resultado houve diferença estatisticamente significativa.

A frequência de consumo alimentar mostrou que $100 \%$ das crianças consumiam arroz. Com relação aos outros alimentos, os resultados mostraram que os mais consumidos diariamente, eram leite e derivados (96,3\%), feijão (79,7\%), frutas $(76,7 \%)$, pães $(62,8 \%)$, manteiga e margarina $(54,6 \%)$ e sucos naturais $(50,1 \%)$.

Foi feita a análise de correlação entre o consumo de alimentos e o IMC para a idade. $\mathrm{O}$ consumo dos alimentos, arroz, feijão, 
pães, macarrão, leite e derivados, salgados, farinhas e achocolatados, apresentou forte correlação com o risco de excesso de peso, excesso de peso e obesidade, sendo que para o excesso de peso os refrigerantes e frituras também apresentaram forte correlação.

\section{DISCUSSÃOO}

Estudos em Salvador e Maceió também verificaram predominância de escolares do sexo feminino sobre o masculino, sendo a correspondência de 54\% e 56,3\%, respectivamente, não mostrando diferença estatisticamente significativa entre o sexo, sendo concordante com o que ocorreu no presente estudo ${ }^{13,14}$. Assim, ao se analisar os dados do presente estudo com os demais mencionados, constatou-se a mesma variação, sempre próximos dos 50\% para ambos os sexos, masculino e feminino, sem ocorrer variação por região do Brasil.

Em Duque de Caxias, Rio de Janeiro, pesquisa realizada com 33 pré-escolares menores de seis anos de idade matriculados em uma creche, a distribuição, segundo o peso atual, apresentou 4 crianças (12\%) entre 22 a $26,2 \mathrm{~kg}, 12$ crianças (36\%) entre 17,4 a $21,4 \mathrm{~kg}$, sendo a maioria, 17 crianças (52\%) na faixa de peso de 11,6 a 16,8 $\mathrm{kg}^{15}$. Ao comparar os dados obtidos nessas duas pesquisas, pode-se observar que, em relação à média de peso atual ocorreu um aumento considerável de $16,8 \mathrm{~kg}$, verifica- do na pesquisa de Duque de Caxias, para $18,43 \mathrm{~kg}$ no presente estudo.

$\mathrm{Na}$ pesquisa de Valente, resultados semelhantes foram obtidos relacionados ao risco de excesso de peso e excesso de peso, $20,51 \%$ e $5,13 \%$, respectivamente, sendo concordante com esta pesquisa. O Brasil, junto à Dinamarca, Itália e Bahvariam, está entre os quatro países que apresentam uma rápida elevação da prevalência de excesso de peso e obesidade em crianças e adolescentes quando avaliados pelo $\mathrm{IMC}^{16}$.

Para se avaliar o consumo de alimentos na escola e no domicílio, nessa pesquisa foi por meio de um Questionário de Frequência de Consumo Alimentar (QFCA). O QFCA é considerado o mais prático e informativo método de avaliação em estudos que investigam a associação entre o consumo dietético e a ocorrência de desfechos clínicos, em geral relacionada às doenças crônicas não transmissíveis ${ }^{17}$.

Em estudos feitos por Triches mostraram a importância do consumo de frutas e hortaliças para a prevenção da obesidade e suas doenças associadas, porém a ingestão destes alimentos em crianças está bem abaixo dos valores recomendados ${ }^{18,19}$.

\section{CONCLUSÕES}

Conclui-se que a prevalência de risco de excesso de peso, excesso de peso e obesidade em pré-escolares foi elevada, porém não

Figura 1- Porcentagem de crianças segundo o sexo em relação ao IMC para Idade.

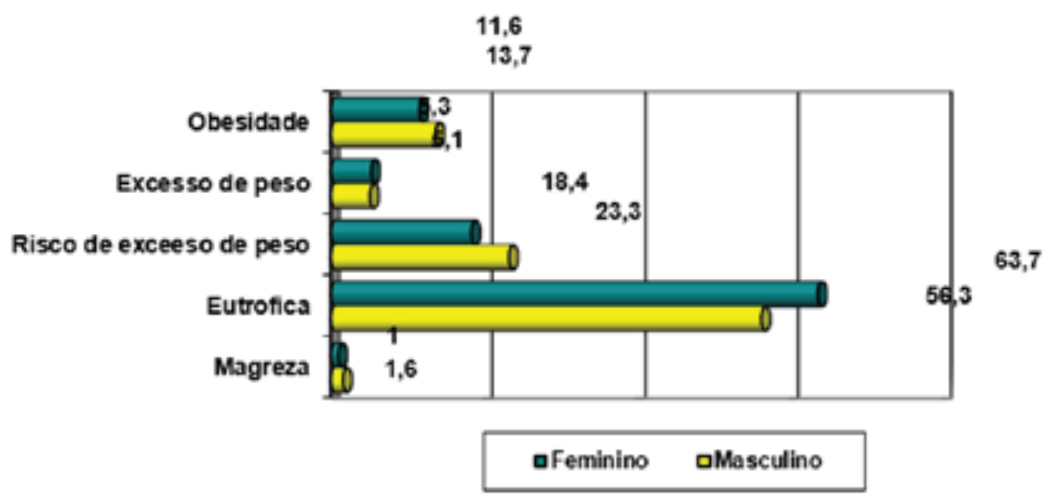

Fonte: Dados da Pesquisa. 
houve diferença significativa $(\mathrm{p}<00,5)$ relacionada ao sexo ou idade dos pré-escolares estudados. No que se refere ao IMC para idade, os resultados mostraram que $60,1 \%$ das crianças estavam eutróficas, 20,8\% com risco de excesso de peso, sendo que 5,2\% tinham excesso de peso e $12,7 \%$ obesidade.

Verificou-se uma forte relação entre o consumo de alimentos fontes de carboidratos e gorduras com o IMC para Idade, no tocante ao risco de excesso de peso, excesso de peso e obesidade.

\section{REFERÊNCIAS}

1. Cunha, NS \& Bonamigo, AW. Prevalence of overcoming and obesity in pré-schools in Brazil: an integrating review. Research, Society and Development. 2020 9(7): 1-21.

2. Kong, AP, Chow CC. Medical consequences of childhood obesity: a Hong Kong perspective. Res Sports Med. 2010; 18:16-25.

3. Falkenbach da Costa L, Malta Fontenele R, Carollo Fernandes MT. Programa Saúde na Escola no Município de Porto Alegre: análise das ações realizadas em 2017. Saúde Coletiva (Barueri) [Internet]. $29^{\circ}$ de setembro de 2020 [citado $11^{\circ}$ de abril de 2021]; 10(56): 3132-41. Disponivel em: http://revistas.mpmcomunicacao.com.br/index.php/saudecoletiva/article/view/900

4. Instituto Brasileiro de Geografia e Estatística (IBGE) Pesquisa de Orçamentos Familiares: 2002-2003. Antropometria e análise do estado nutricional de crianças e adolescentes no Brasil. Rio de Janeiro: IBGE; 2006.

5. Instituto Brasileiro de Geografia e Estatística (IBGE). Pesquisa de orçamentos familiares 2008-2009: antropometria e estado nutricional de crianças, adolescentes e adultos no Brasil. Rio de Janeiro: IBGE; 2010.

6. Longo-Silva, G. Toloni, M. H. A. Goulart, R. M. M. Taddei, J. A. C. Avaliação do consumo alimentar em creches públicas em São Paulo, Brasil. Revista Paulista de Pediatria, Recife, v. 30, n. 1, p. 35-41, 2012

7. Moreira-araújo, R. S. R, Araújo, M. A. M, Silva, A. M. S, Carvalho, C. M. R, Arêas, J. A. G. Impacto de Salgadinho de alto Valor Nutritivo na Situação Nutricional de Creches Municipais de Teresina - PI. Nutrire Revista da Sociedade Brasileira de Alimentação Nutrição Journal Brasilian Food Nutricion, São Paulo:, v23, n15, p.7-21, 2002.

8. Silva, Sara Araújo da, Moura, Erly Catarina de. Determinantes do estado de saúde de crianças ribeirinhas menores de dois anos de idade do Estado do Pará, Brasil: um estudo transversal. Cadernos de Saúde Pública, Rio de Janeiro, v. 26, n. 2, p.273-285, fev, 2010. World Health Organization (WHO).

9. World Health Estatistic, 2012. Disponível em: http://www. who.int/gho/publications/world_health_statistics/2012/ en/.

10. Cole, T.J.; Bellizzi, M.C.; Flegal, K. M.; Dietz, W. H. - Establishing a standard definition for child overweight and obesity worldwide: international survey. British Medical Journal-, BMJ. v. 320, p. 1-6, 2000.

11. Brandão; Socorro Adriana de Sousa Meneses. Excesso de peso, obesidade e consumo alimentar em pré-escolares de Teresina-PI. Dissertação (Mestrado em Ciência e Saúde)-Uni- versidade Federal do Piauí. Teresina, 2014.

12. Andrade, D. F, Ogilari, P. J. Estatística para as ciências agrárias e biológicas: com noções de experimentação. Florianópolis: Ed. da UFSC, p. 470, 2010.

13. Boa-Sorte Ney, Neri Luciana A., Leite Maria Efigênia Q., Brito Sheila M., Meirelles Ana Regina, Luduvice Fábia B. S. et al. Maternal perceptions and self-perception of the nutritional status of children and adolescents from private schools. J. Pediatr. (Rio J.) [Internet]. 2007 Ago [citado 2021 Abr 30] ; 83( 4 ): 349-356. Disponível em: http://www.scielo.br/scielo.php?script=sci_arttext\&pid=S0021-75572007000500011\&lng=pt. http://dx.doi. org/10.2223/JPED.1678.

14. Mendonça Maria Roseane Tenorio, Silva Maria Alayde Mendonça da, Rivera Ivan Romero, Moura Adriana Ávila. Prevalência de sobrepeso e obesidade em crianças e adolescentes da cidade de Maceió. Rev. Assoc. Med. Bras. [Internet]. 2010 [cited 2021 Apr 30] ; 56( 2 ): 192-196. Available from: http://www.scielo.br/scielo.php script=sci_ arttext\&pid=S0104-42302010000200018\&Ing=en. http:// dx.doi.org/10.1590/S0104-42302010000200018.

15. Santos, A. L. B, Leão, L. S. C. S. Perfil Antropométrico de pré-escolares de uma creche em Duque de Caxias, Rio de Janeiro. Revista Paulista de Pediatria 2008, 26 (3): 218-24.

16. Valente, T. B.; Hecktheuer, L. H. R.; Brasil, C. C. B. Socioeconomic conditions, food intake and nutritional status of preschool children belonging to a day care center. Alimentos e Nutrição-Araraquara, v. 21, n. 3, p. 421-428, 2010.

17. Fisberg Regina Mara, Marchioni Dirce Maria Lobo, Colucci Ana Carolina Almada. Avaliação do consumo alimentar e da ingestão de nutrientes na prática clínica. Arq Bras Endocrinol Metab [Internet]. 2009 July [cited 2021 Apr 30]; 53( 5 ): 617624. Available from: http://www.scielo.br/scielo.php?script=sci_arttext\&pid=S0004-27302009000500014\&Ing=en. https://doi.org/10.1590/S0004-27302009000500014.

18. Trinches. R. M, Giugliani E.R. Obesity, eating habits and nutritional knowledge among school children. Revista de Saúde Pública, v.39, p541-547, 2005.

19. Mon Mondini Lenise, Levy Renata Bertazzi, Saldiva Silvia Regina Dias Médici, Venâncio Sonia Isoyama, Aguiar Jeanice de Azevedo, Stefanini Maria Lúcia Rosa. Overweight, obesity and associated factors in first grade schoolchildren in a city of the metropolitan region of São Paulo, Brasil. Cad. Saúde Pública [Internet]. 2007 Aug [cited 2021 Apr 30] ; 23(8): 1825-1834. Available from: http://www.scielo.br/scielo.php?script=sci_ arttext\&pid=S0102-311X2007000800009\&Ing=en. https:// doi.org/10.1590/S0102-311X2007000800009. 